\title{
Sphingobacterium spiritivorum bacteremia due to cellulitis in an elderly man with chronic obstructive pulmonary disease and congestive heart failure: a case report
}

\author{
Arata Hibi ${ }^{i^{*}}$ and Yuka Kumano ${ }^{2}$
}

\begin{abstract}
Background: Sphingobacterium spiritivorum is a glucose non-fermenting Gram-negative rod, formerly classified as one of the Flavobacterium species. It is characterized by a large number of cellular membrane sphingophospholipids. Sphingobacterium species are ubiquitous and isolated from natural environments, such as soil and water. However, they rarely cause infections in humans. Only a limited number of cases have been reported in elderly and immunocompromised patients with underlying diseases and predisposing factors.

Case presentation: An 80-year-old Japanese man with chronic obstructive pulmonary disease and congestive heart failure visited the Kariya Toyota General Hospital, Aichi, Japan with the chief complaint of fever accompanied by chills and left leg pain. At initial presentation, he was distressed and dyspneic. He was febrile $\left(38.8^{\circ} \mathrm{C}\right)$, and his left foot was swollen with reddening and tenderness. We diagnosed him as having cellulitis, and he was hospitalized for antibiotic therapy. Initially, he was treated with intravenously administered cefazolin, but after the isolation of a glucose nonfermenting Gram-negative rod from blood cultures, we decided to switch cefazolin to intravenously administered meropenem on day 4, considering the antibiotic resistance of the causative organism. The causative organism was identified as S. spiritivorum on day 6 . His condition gradually stabilized after admission. Meropenem was switched to orally administered levofloxacin on day 12 . He was discharged on day 16 and treated successfully without any complications.
\end{abstract}

Conclusions: Although S. spiritivorum is rare, with limited cases isolated from cellulitis, it should be considered as a causative organism in elderly and immunocompromised patients with cellulitis. Blood cultures are the key to correct diagnosis and appropriate treatment.

Keywords: Sphingobacterium spiritivorum, Cellulitis, Bacteremia

\section{Background}

Sphingobacterium spiritivorum (S. spiritivorum) is a glucose non-fermenting Gram-negative rod (GNF-GNR), formerly classified as one of the Flavobacterium species [1]. It is characterized by a large number of cellular membrane sphingophospholipids [1]. Sphingobacterium species are ubiquitous and isolated from natural environments, such as soil and water. However, they rarely

\footnotetext{
* Correspondence: exchange1113@yahoo.co.jp

'Division of Nephrology and Rheumatology, Department of Internal

Medicine, Kariya Toyota General Hospital, 5-15, Sumiyoshi-cho, Kariya, Aichi

448-8505, Japan

Full list of author information is available at the end of the article
}

cause infection in humans. Only a limited number of cases have been reported in elderly and immunocompromised patients with underlying disease and predisposing factors [2-6]. However, S. spiritivorum has the potential of causing fatal infections and bacteremia, particularly in elderly and immunocompromised patients. Although our case is not the first case report of $S$. spiritivorum infection isolated from humans, we propose that it is important to consider S. spiritivorum as a causative organism in selected patients with cellulitis. 


\section{Case presentation}

An 80-year-old Japanese man presented to our hospital with complaints of fever and left leg pain, as well as bilateral lower extremity swelling. Although mild edema was always observed in his bilateral lower extremities, it gradually worsened 1 week prior to admission. On the day of admission, he had high fever accompanied by chills. He denied any recent leg trauma. His past medical history was significant for pulmonary tuberculosis at 30 years of age, chronic obstructive pulmonary disease (COPD), and congestive heart failure (CHF). A pacemaker was inserted because of atrial fibrillation (AF) with symptomatic bradycardia. He was an ex-tobacco smoker (100 pack year history) and did not drink alcohol. His maintenance medications were dabigatran (220 $\mathrm{mg}$ ), furosemide $(20 \mathrm{mg})$, and an inhaled corticosteroid/ long-acting $\beta_{2}$-agonist.

At initial presentation, he was alert and oriented but appeared distressed. His vital signs were as follows: body temperature, $38.8{ }^{\circ} \mathrm{C}$; blood pressure, 135/90 mmHg; heart rate, 96 beats per minute with irregular rhythm; blood oxygen saturation, 93\% with room air; and respiratory rate, 22 breaths per minute. He had a barrel-shaped chest, coarse crackles were heard over the lung base, and wheezes were heard over both lung fields. Pitting edema was observed in his lower extremities. His left foot had more erythema and edema than his right foot (Fig. 1). His left lower extremity was warm and tender to touch. There were no skin breaks or other potential infection entry sites. Tinea pedis was ascertained by a potassium hydroxide test.

Arterial blood gas analysis showed the following results: $\mathrm{pH}, 7.408$; partial pressure of carbon dioxide, 44 mmHg; partial pressure of oxygen, $72 \mathrm{mmHg}$; bicarbonate, $22.5 \mathrm{mmol} / \mathrm{L}$; and lactate, $1.8 \mathrm{mmol} / \mathrm{L}$. A complete blood count revealed the following results: white blood cells, 9,000/ $\mu \mathrm{L}$; red blood cells, $384 \times 10^{4} /$ $\mu \mathrm{L}$; hemoglobin, $12.4 \mathrm{~g} / \mathrm{dL}$; and platelets, $9.6 \times 10^{4} / \mu \mathrm{L}$. Chemistry results were as follows: serum creatinine, 0.81 $\mathrm{mg} / \mathrm{dL}$; blood urea nitrogen, $19.0 \mathrm{mg} / \mathrm{dL}$; albumin, $3.7 \mathrm{~g} /$ $\mathrm{dL}$; total bilirubin, $2.1 \mathrm{mg} / \mathrm{dL}$; aspartate aminotransferase, $43 \mathrm{U} / \mathrm{L}$; alanine aminotransferase, $22 \mathrm{U} / \mathrm{L} ; \mathrm{C}$ reactive protein, $0.36 \mathrm{mg} / \mathrm{dl}$; and brain natriuretic peptide (BNP), $471 \mathrm{pg} / \mathrm{mL}$ (our patient's basal BNP level was approximately $100 \mathrm{pg} / \mathrm{mL})$. A coagulation test showed prolonged prothrombin time-international normalized ratio (1.29) and activated partial thromboplastin time (52.3 seconds). A chest X-ray showed a nodular lesion on the right pulmonary apex compatible with previous tuberculous infection without any infiltrations. An electrocardiogram showed AF with pacemaker rhythm without any sensing and pacing failures. Based on these results, we diagnosed our patient as having cellulitis and immediately admitted him to hospital because his respiratory status continued to worsen, necessitating supplemental oxygen therapy for dyspnea relief. We also considered a risk of sepsis and mortality because his sequential organ failure assessment score was 5 points ( 2 points greater than baseline) at initial presentation [7].

Cefazolin ( $1 \mathrm{~g}$ every 8 hours) was initially administered intravenously to treat his cellulitis, but after 25 hours of incubation, two sets of aerobic blood culture bottles (BD BACTEC $^{\text {tix }}$ Plus Aerobic/F Medium; BD Diagnostics, Sparks, MD, USA) were found to be positive (detected by BD BACTEC ${ }^{\mathrm{m}} \mathrm{FX}$, Blood Culture System; BD Diagnostics, Sparks, MD, USA) for GNRs (Fig. 2). The positive culture broth was inoculated onto a blood agar plate (BD BBL ${ }^{\mathrm{m}}$ Trypticase $^{\text {Tn }}$ Soy Agar with 5\% Sheep Blood; Nippon
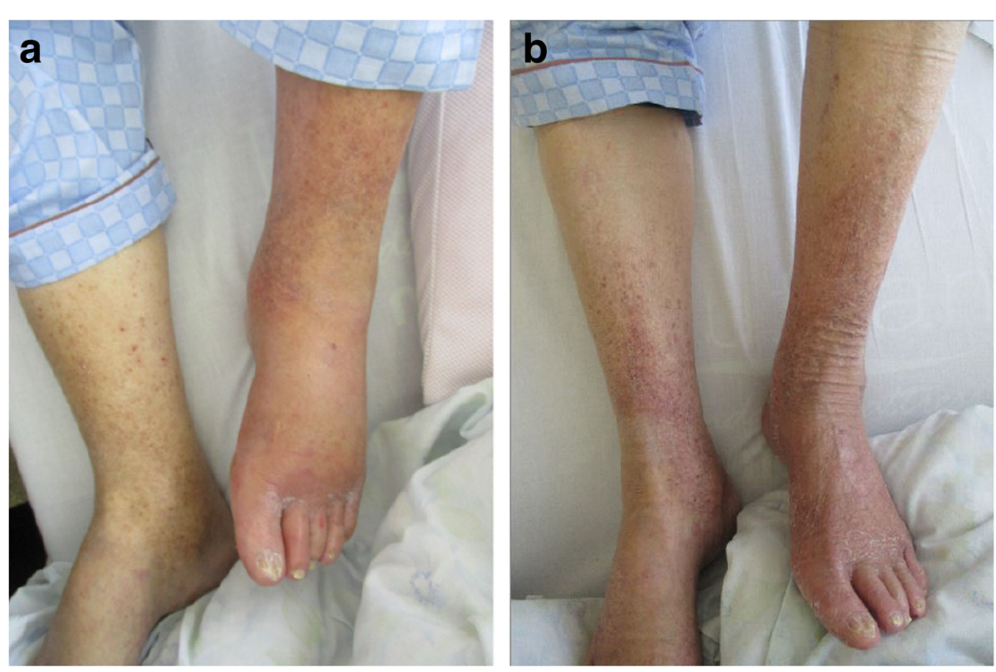

Fig. 1 Appearance of lower extremities of the patient on the day of admission (a) and after treatment (b). On the day of admission, reddening and swelling were observed on the left leg. Dermatophyte was positive in the toe webs as per a potassium hydroxide test 


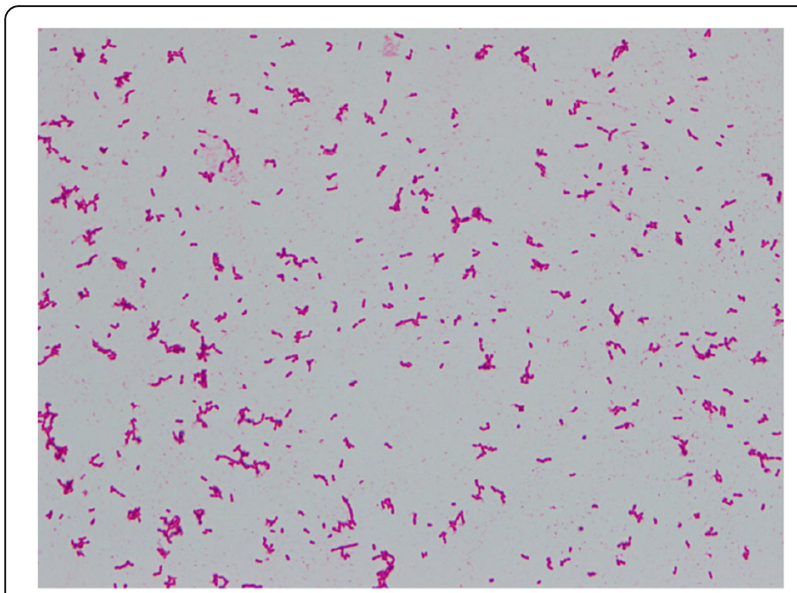

Fig. 2 Gram stain of the organism isolated from blood culture (magnification, ×1000). Gram-negative short rods were seen

Becton Dickinson Company, Fukushima, Japan) and light yellow colonies were observed after incubation of 24 hours at $37^{\circ} \mathrm{C}$. The causative organism was confirmed as GNF-GNR on day 4. Accordingly, we switched antibiotics to intravenously administered meropenem ( 1 g every 8 hours) on the same day, considering antibiotic resistance. On day 6, the causative organism was identified as S. spiritivorum. It was identified by BD PHOENIX ${ }^{\mathrm{mi}}$ System (BD Diagnostics, Sparks, MD, USA) and matrix-assisted laser desorption/ionization time of flight mass spectrometry, using Microflex LT with MALDI Biotyper version 3.1 database (Bruker Daltonik, Bremen, Germany). Our patient's condition gradually improved with the antibiotic use. We decided to switch antibiotics to orally administered levofloxacin (500 $\mathrm{mg} /$ day) on day 12 , considering the antibiotic sensitivity of S. spiritivorum isolated from the blood culture (Table 1). Trimethoprim/sulfamethoxazole was an alternative but was not used out of concern for adverse drug reactions considering our patient's age. He was discharged on day 16 without any complications, and the antibiotic was discontinued on the same day. We followed up with him 2 weeks after discharge, during which he did not have any residual symptoms related to cellulitis.

\section{Discussion}

Sphingobacterium species are aerobic, Gram-negative, short rod, non-motile, non-spore-forming bacteria. They are oxidase-positive, catalase-positive, and ureasepositive and indole-negative and produce light yellow colonies on blood agar plates [1]. Thus far, more than 20 species in the genus Sphingobacterium have been reported based on $16 \mathrm{~S}$ ribosomal ribonucleic acid gene sequencing [8] and the number of isolated species is increasing. S. spiritivorum was first isolated from a human clinical specimen by Holmes et al. in 1982 [9] and was
Table 1 Antibiotics sensitivity of Sphingobacterium spiritivorum isolated from the present case

\begin{tabular}{lll}
\hline Antibiotics & $\begin{array}{l}\text { Minimal inhibitory concentration } \\
(\mu \mathrm{g} / \mathrm{mL})\end{array}$ & Sensitivity \\
\hline Piperacillin & 16 & $\mathrm{~S}$ \\
Cefoperazone & 32 & $\mathrm{I}$ \\
Ceftazidime & $\leq 4$ & $\mathrm{~S}$ \\
Cefepime & 4 & $\mathrm{~S}$ \\
Latamoxef & $>32$ & $\mathrm{R}$ \\
Imipenem/cilastatin & $\leq 1$ & $\mathrm{~S}$ \\
Meropenem & $\leq 1$ & $\mathrm{~S}$ \\
Piperacillin/tazobactam & 8 & $\mathrm{~S}$ \\
Aztreonam & $>16$ & $\mathrm{R}$ \\
Amikacin & $>32$ & $\mathrm{R}$ \\
Tobramycin & $>8$ & $\mathrm{R}$ \\
Gentamycin & $>8$ & $\mathrm{R}$ \\
Minocycline & $\leq 1$ & $\mathrm{~S}$ \\
Trimethoprim/ & $\leq 20$ & $\mathrm{~S}$ \\
sulfamethoxazole & & $\mathrm{S}$ \\
Levofloxacin & $\leq 1$ & $\mathrm{~S}$ \\
Ciprofloxacin & $\leq 0.5$ & \\
\hline
\end{tabular}

$I$ intermediate, $R$ resistant, $S$ susceptible

initially described as Flavobacterium spiritivorum. In 1983, Yabuuchi et al. first proposed Sphingobacterium as a new genus [10]. The genus Sphingobacterium differs from the genus Flavobacterium by high cellular membrane concentrations of sphingophospholipid and ceramide. Naka et al. performed a structural analysis of sphingophospholipids in S. spiritivorum, thereby purifying a novel sphingolipid among eukaryotic and prokaryotic cells [11].

Sphingobacterium species are ubiquitous and commonly isolated from soil, plants, and water, but rarely from human infection sites. Sphingobacterium multivorum and S. spiritivorum were isolated from very few existing cases. Lambiase et al. reported the isolation of S. multivorum and S. spiritivorum from the sputum of patients with cystic fibrosis [12]. Recently, the first human case of Sphingobacterium hotanense infection in an elderly patient was reported [13]. In that case, scratches on the right arm caused by a rooster were the suspected infection entry site from soil.

Sphingobacterium species are resistant to commonly used antibiotics [1]. S. multivorum can produce an extended-spectrum $\beta$-lactamase and a metallo- $\beta$ lactamase, which make it resistant to third-generation cephalosporins and carbapenems, respectively [14]. S. spiritivorum is susceptible to carbapenems. Quinolones, trimethoprim-sulfamethoxazole, and ceftazidime are effective in vitro, which is compatible with previous clinical 
reports [12]. S. spiritivorum isolated from the present case was susceptible to the antibiotics listed above. In the present case, we observed a good clinical course with intravenously administered meropenem followed by orally administered levofloxacin.

We identified five previously reported cases of S. spiritivorum infection in the English literature [2-6] (Table 2). Three cases were caused by cellulitis $[2,3,6]$ and two cases by catheter-related blood stream infection $[4,5]$. In most of these cases, the patients had predisposing factors and underlying diseases, such as Parkinson's disease (with chronic venous stasis due to akinesia and injuries from frequent falls, which are risk factors for cellulitis) $[2,3]$, refractory anemia [4], acute myeloid leukemia treated with chemotherapy [5], and end-stage renal disease on hemodialysis [6]. One case of extrinsic allergic alveolitis (hypersensitivity pneumonitis) caused by $S$. spiritivorum [15] was not included because it was not a direct infection but was caused by a hypersensitivity reaction against organism-derived allergens [16]. In our case, edema due to CHF was a risk factor for cellulitis [17]. Aging and COPD can also increase susceptibility to infections $[18,19]$. Tinea pedis is a risk factor for cellulitis [20] because it may provide entry sites for infections [21] and changes in bacterial flora [22].

Although obtaining blood cultures of patients with cellulitis may not be cost effective, given the low rate of positive blood cultures (2.0\%) [23], we could not have made a correct diagnosis in the present case without blood cultures. Mills and Chen reviewed several studies and concluded that obtaining blood cultures does not significantly alter treatment or aid in diagnosing the causative organism in immunocompetent patients with acute cellulitis [24]. In addition, the current Infectious Diseases Society of America (IDSA) guidelines do not recommend routine performance of blood cultures in patients with cellulitis; however, performing blood cultures is recommended in patients with malignancy, chemotherapy, neutropenia, severe cell-mediated immunodeficiency, immersion injuries, and animal bites [25]. Peralta et al. reported the absence of previous antibiotic treatment and the presence of two or more comorbid factors including obesity, COPD, diabetes, alcohol addiction, liver cirrhosis, CHF, and immunocompromised condition were associated with bacteremia in patients with cellulitis [26]. Lee et al. proposed an initial diagnostic prediction model with four independent predictors for estimating probability of bacteremia in patients with cellulitis: age $\geq 65$ years, involvement of non-lower extremities, liver cirrhosis, and systemic inflammatory response syndrome [27]. In a recent study, van Daalen et al. reported the blood culture positivity rate was higher than the rates reported by IDSA guidelines in hospitalized patients with skin and soft tissue infections, particularly in patients with severe comorbidity [28]. Evaluation of patients' comorbidity is critical to making decisions to perform blood cultures in patients with cellulitis. Considering $S$. spiritivorum was isolated from blood cultures in all of the previous reports, performing blood cultures in patients with cellulitis with comorbid risk factors can be useful to identify the causative organism and important for appropriate treatment.

\section{Conclusions}

S. spiritivorum is a rare causative organism of cellulitis, with a limited number of reported cases in the literature. In the present case, aging and COPD could have been the risk factors for infection, and edema due to $\mathrm{CHF}$ was a predisposing factor for cellulitis. Tinea pedis could have produced an infection entry site. Although our patient was initially septic, he was successfully treated by administration of targeted antibiotics. Blood cultures were key to identifying the causative organism in the present case. We should consider S. spiritivorum as a

Table 2 Previously reported five cases of Sphingobacterium spiritivorum infections and the present case

\begin{tabular}{|c|c|c|c|c|c|c|c|}
\hline $\begin{array}{l}\text { Case number } \\
\text { and Reference }\end{array}$ & $\begin{array}{l}\text { Reported } \\
\text { year }\end{array}$ & $\begin{array}{l}\text { Age/ } \\
\text { Sex }\end{array}$ & $\begin{array}{l}\text { Underlying diseases and predisposing } \\
\text { factors }\end{array}$ & $\begin{array}{l}\text { Source of } \\
\text { isolation }\end{array}$ & Diagnosis & Antibiotics & $\begin{array}{l}\text { Clinical } \\
\text { outcome }\end{array}$ \\
\hline Case 1 [2] & 2002 & $72 / \mathrm{M}$ & Parkinson's disease & Blood & Cellulitis & $\begin{array}{l}\text { Cefazolin followed by } \\
\text { ampicillin/sulbactam }\end{array}$ & $\begin{array}{l}\text { Complete } \\
\text { recovery }\end{array}$ \\
\hline Case 2 [3] & 2003 & $84 / \mathrm{M}$ & Refractory anemia & Blood & Cellulitis & Amoxicillin/clavulanate & $\begin{array}{l}\text { Complete } \\
\text { recovery }\end{array}$ \\
\hline Case $3[4]$ & 2013 & $68 / F$ & $\begin{array}{l}\text { Acute myeloid leukemia treated with } \\
\text { chemotherapy }\end{array}$ & Blood & CRBSI & $\begin{array}{l}\text { Cefepime followed by } \\
\text { ciprofloxacin }\end{array}$ & Died \\
\hline Case $4[5]$ & 2016 & $80 / F$ & $\begin{array}{l}\text { ESRD on hemodialysis via tunneled } \\
\text { central venous dialysis catheter; DM }\end{array}$ & Blood & CRBSI & $\begin{array}{l}\text { Trimethoprim followed by } \\
\text { meropenem and ciprofloxacin }\end{array}$ & $\begin{array}{l}\text { Complete } \\
\text { recovery }\end{array}$ \\
\hline Case $5[6]$ & 2016 & $89 / \mathrm{M}$ & $\begin{array}{l}\text { Parkinson's disease; skin tears and } \\
\text { abrasion due to multiple falls }\end{array}$ & Blood & Cellulitis & $\begin{array}{l}\text { Piperacillin/tazobactam followed } \\
\text { by amoxicillin/clavulanate }\end{array}$ & $\begin{array}{l}\text { Complete } \\
\text { recovery }\end{array}$ \\
\hline The present case & 2017 & $80 / \mathrm{M}$ & COPD; edema due to CHF; tinea pedis & Blood & Cellulitis & $\begin{array}{l}\text { Meropenem followed by } \\
\text { levofloxacin }\end{array}$ & $\begin{array}{l}\text { Complete } \\
\text { recovery }\end{array}$ \\
\hline
\end{tabular}

CHF congestive heart failure, COPD chronic obstructive pulmonary disease, CRBSI catheter-related blood stream infection, DM diabetes mellitus, ESRD end-stage renal disease, $F$ female, $M$ male 
potential causative organism of cellulitis, particularly in patients with comorbid risk factors.

\section{Abbreviations}

AF: Atrial fibrillation; BNP: Brain natriuretic peptide; CHF: Congestive heart failure; COPD: Chronic obstructive pulmonary disease; GNF-GNR: Glucose non-fermenting Gram-negative rod; IDSA: Infectious Diseases Society of America

\section{Acknowledgements}

We are grateful to the clinical microbiological technicians of Kariya Toyota General Hospital who support our daily clinical practice.

\section{Funding}

The authors declare that there is no funding related to this manuscript.

\section{Availability of data and materials}

The datasets used and/or analyzed during the current study are available from the corresponding author on reasonable request.

\section{Authors' contributions}

$\mathrm{AH}$ is responsible for the manuscript. YK contributed to the critical revision of the manuscript and supported the clinical management of the patient. Both authors read and approved the final version of the manuscript.

\section{Ethics approval and consent to participate}

Based on the Ethical Guidelines for Medical and Health Research involving Human Subjects in Japan, ethics approval is not necessary when submitting case reports.

\section{Consent for publication}

Written informed consent was obtained from the patient for publication of this case report and any accompanying images. A copy of the written consent is available for review by the Editor-in-Chief of this journal.

\section{Competing interests}

The authors declare that they have no competing interests.

\section{Publisher's Note}

Springer Nature remains neutral with regard to jurisdictional claims in published maps and institutional affiliations.

\section{Author details}

'Division of Nephrology and Rheumatology, Department of Internal Medicine, Kariya Toyota General Hospital, 5-15, Sumiyoshi-cho, Kariya, Aichi 448-8505, Japan. ²Department of Dermatology, Kariya Toyota General Hospital, 5-15, Sumiyoshi-cho, Kariya, Aichi 448-8505, Japan.

Received: 14 June 2017 Accepted: 6 September 2017

Published online: 30 September 2017

\section{References}

1. Steinberg JP, Burd EM. Other Gram-Negative and Gram-Variable Bacilli. In: Benett JE, Dolin R, Blazer MJ, editors. Mandell, Douglas, and Bennett's Principals and Practices of Infectious Disease. 8th ed. Philadelphia: Saunders; 2015. p. 2680-1.

2. Marinella MA. Cellulitis and sepsis due to Sphingobacterium. JAMA 2002;16:1985

3. Tronel $\mathrm{H}$, Plesiat $\mathrm{P}$, Ageron $\mathrm{E}$, Grimont PA. Bacteremia caused by a novel species of Sphingobacterium. Clin Microbiol Infect. 2003;12:1242-4.

4. Koh YR, Kim SY, Chang CL, Shin HJ, Kim KH, Yi J. The first Korean case of Sphingobacterium spiritivorum bacteremia in a patient with acute myeloid leukemia. Ann Lab Med. 2013:4:283-7.

5. Gupta A, Logan J, Elhag N, Almond M. Sphingobacterium spiritivorum infection in a patient with end stage renal disease on hemodialysis. Ann Clin Microbiol Antimicrob. 2016. doi:10.1186/s12941-016-0141-5.

6. Anthony JM, Verma R. Sphingobacterium spiritivorum septicemia associated with cellulitis in a patient with Parkinson's disease. BMJ Case Rep. 2016. doi: 10.1136/bcr-2016-215319.
7. Singer M, Deutschman CS, Seymour CW, Shankar-Hari M, Annane D, Bauer M, et al. The Third International Consensus Definitions for Sepsis and Septic Shock (Sepsis-3). JAMA. 2016:8:801-10.

8. Sun JQ, Liu M, Wang XY, Xu L, Wu XL. Sphingobacterium suaedae sp. nov, isolated from the rhizosphere soil Suaeda corniculata. Int J Syst Evol Microbiol. 2015;12:4508-13.

9. Holmes B, Owen RJ, Hollis DG. Flavobacterium spiritivorum, a new species isolated from human clinical specimens. Int J Syst Bacterial. 1982;2:157-65.

10. Yabuuchi E, Kaneko T, Yano I, Moss CW, Miyoshi N. Sphingobacterium gen. nov., Sphingobacterium spiritivorum comb. nov., Sphingobacterium multivorum comb. nov., Sphingobacterium mizutae sp. nov., and Flavobacterium indologenes sp. nov.: glucose-nonfermenting gram-negative rods in CDC group IIK-2 and IIb. Int J Syst Bacteriol. 1983;3:580-98.

11. Naka T, Fujiwara N, Yano I, Maeda S, Doe M, Minamino M, et al. Structural analysis of sphingophospholipid derived from Sphingobacterium spiritivorum, the type species of genus Sphingobacterium. Biochem Biophy Acta. 2003:2-3:83-92.

12. Lambiase A, Rossano F, Del Pezzo M, Raia V, Sepe A, de Gregorio F, et al. Sphingobacterium respiratory tract infection in patient with cystic fibrosis. BMC Res Notes. 2009. doi:10.1186/1756-0500-2-262.

13. Kroumova V, Rossati A, Bargiacchi O, Garavelli PL, Camaggi A, Caroppo S, et al. From soil to blood: first human case of Sphingobacterium hotanense bacteremia. Infez Med. 2017;1:75-6

14. Blahová Králiková K, Krcméry Sr V, Kubonová K. Hydrolysis of imipenem, meropenem, ceftazidime, and cefepime by multiresistant nosocominal strains of Sphingobacterium multivorum. Eur J Clin Microbiol Infect Dis. 1997:2:178-80.

15. Kämpfer $P$, Engelhart $S$, Rolke $M$, Sennekamp J. Extrinsic allergic alveolitis (hypersensitivity pneumonitis) caused by Sphingobacterium spiritivorum from the water reservoir of steam iron. J Clin Microbiol. 2005;9:4908-10.

16. Riario Sforza GG, Marinou A. Hypersensitivity pneumonitis: a complex lung disease. Clin Mol Allergy. 2017. doi:10.1186/s12948-017-0062-7.

17. Conway EL, Sellick JA, Kurtzhalts K, Mergenhagen KA. Obesity and heart failure as predictors of failure in outpatient skin and soft tissue infections. Antimicrob Agents Chemother. 2017. doi:10.1128/AAC.02389-16.

18. Montecino-Rodriguez E, Berent-Maoz B, Dorshkind K. Causes, consequence, and reversal of immune system aging. J Clin Invest. 2013:3:958-65.

19. Wilkinson TMA. Immune checkpoints in chronic obstructive lung disease. Eur Respir Rev. 2017. doi:10.1183/16000617.0045-2017.

20. Bristow IR, Spruce MC. Fungal foot infection, cellulitis and diabetes: a review. Diabet Med. 2009:5:548-51.

21. Björnsdóttir S, Gottfredsson M, Thórisdóttir AS, Gunnarsson GB, Ríkardsdóttir $\mathrm{H}$, Kristjánsson M, et al. Risk factors for acute cellulitis of the lower limb: a prospective case-control study. Clin Infect Dis. 2005:10:1416-22.

22. Leyden JJ, Kligman AM. Interdigital athlete's foot. The interaction of dermatophytes and resident bacteria. Arch Dermatol. 1978;10:1466-72.

23. Perl B, Gottehrer NP, Raveh D, Schlesinger Y, Rudensky B, Yinnon AM. Cost-effectiveness of blood cultures for adult patients with cellulitis. Clin Infect Dis. 1999;6:1483-8.

24. Mills $\mathrm{AM}$, Chen $\mathrm{EH}$. Are blood cultures necessary in adults with cellulitis? Ann Emerg Med. 2005;5:548-9.

25. Stevens DL, Bisno AL, Chambers HF, Dellinger EP, Goldstein EJ, Gorbach SL, et al. Practice guidelines for the diagnosis and management of skin and soft tissue infections: 2014 update by the Infectious Diseases Society of America. Clin Infect Dis. 2014;2:e10-52

26. Peralta G, Padrón E, Roiz MP, De Benito I, Garrido JC, Talledo F, et al. Risk factors for bacteremia in patients with limb cellulitis. Eur J Clin Microbiolo Infecti Dis. 2006;10:619-26.

27. Lee CY, Kunin CM, Chang C, Lee SS, Chen YS, Tsai HC. Development of a prediction model for bacteremia in hospitalized adults with cellulitis to aid in the efficient use of blood cultures: a retrospective cohort study. BMC Infec Dis. 2016. doi:10.1186/s12879-016-1907-2

28. Van Daalen FV, Kallen MC, van den Bosch CMA, Hulscher MEJL, Geerlings SE, Prins JM. Clinical condition and comorbidity as determinants for blood culture positivity in patients with skin and soft-tissue infections. Eur J Clin Microbiol Infect Dis. 2017. doi:10.1007/s10096-017-3001-0. 